\title{
AI Safety and General Collective Intelligence
}

\author{
Andy E. Williams ${ }^{1, *}$ \\ ${ }^{1}$ Nobeah Foundation, Nairobi, Kenya
}

\begin{abstract}
Considering both current narrow AI, and any Artificial General Intelligence (AGI) that might be implemented in the future, there are two categories of ways such systems might be made safe for the human beings that interact with them. One category consists of mechanisms that are internal to the system, and the other category consists of mechanisms that are external to the system. In either case, the complexity of the behaviours that such systems might be capable of can rise to the point at which such measures cannot be reliably implemented. However, General Collective Intelligence or GCI can exponentially increase the general problem-solving ability of groups, and therefore their ability to manage complexity. This paper explores the specific cases in which AI or AGI safety cannot be reliably assured without GCI.
\end{abstract}

Keywords: General Collective Intelligence, Human-Centric Functional Modelling, AI safety, AGI safety

\section{Background}

General Collective Intelligence or GCI has been defined as a platform that combines individuals into a single intelligence with the potential for exponentially greater general problem-solving ability (intelligence) than any individual.

\section{Introduction}

Virtually all work on AI safety has focused on how to control AI through various top down internal or external means according to what is right or wrong by some AI researcher, or AI research donor's perspective [1], as opposed to bottom up methods that relies on some mechanism for "calculating" or "observing" what is ethical. Whether ensuring AI is employed and operates in an "ethical" way, or whether ensuring AI doesn't suggest harmful solutions such as to eliminate all unhealthy people instead of finding better treatment methods when asked to find measures to maximize the health of a population, such approaches might be missing two fundamental truths.

Firstly, from a functional modeling perspective the cognition traces a path through a "conceptual space" as it executes reasoning. A problem is then the lack of a path from one concept to another. The level of general problem-solving ability (intelligence) is then the volume of conceptual space that can be navigated per unit time. The increase in problem-solving ability that might be achieved with a tool such as AI that has narrow problem-solving ability can be conceptualized as increasing intelligence by the volume of a narrow rod in conceptual space. This narrow rod represents increased problem-solving ability in the narrow topic chosen by the programmer. Wherever AI must be redesigned by a human in order to target a different problem, the problemsolving process is again limited by the human programmer's innate conceptual space and its ability to redirect that narrow cone of AI problemsolving ability into a different direction through 
redesign of the AI. Once the AI is redesigned, that narrow rod can be extended in a different direction. But as the number of AI solutions increases, they potentially become unmanageable for the human mind. Even if that narrow rod were to be extended in all directions, the human conceptual space at the centre of that volume would be its weakness. That human mind would have to find those AI solutions in order to reuse them. The general problem-solving ability (intelligence) of the human with its library of AI solutions would not be as great as that of an AGI with the ability to integrate all of those AI solutions into a single reasoning process without the bottleneck of the human mind.

This functional modeling perspective clarifies that AI is a tool, so that the issue of AI safety can be clarified as ensuring the tool operates within safe parameters, as well as ensuring humans use this tool within those parameters. An Artificial General Intelligence (AGI) however is an actor rather than a tool. Just as people make nondeterministic choices, this functional modeling perspective clarifies that an AGI will make its own non-deterministic choices as well. Rather than being deterministically controllable, with an independent actor one can only try to understand all potential uses, and optimize outcomes of safe tool use as well as minimize the consequence of unsafe tool use through design of the tool and through design of interventions that help ensure the actor uses the tool within safe parameters. One might optimize the degree to which tool use is constrained to safe parameters, as well as minimizing potential risks of the tool being used outside safe parameters, through whatever means can be imposed on that actor, whether through methods of coercion, control, or through whatever incentives that exist and that assure critical benefits, and maximize other non-critical benefits, as well as that eliminate critical risks, and minimize other risks, Creating the capacity to introduce such measures requires the capacity to model and assess potential human or AGI reasoning, and to assess the measures that might be used to guide that reasoning, and to do all of this at the exponentially higher rate and scale AGI might be likely to achieve, or at the exponentially higher rate and scale required to govern all human developers of AI.

The second truth is that different fundamental cognitive biases determine how individuals assess truth. These biases are potentially irreconcilable and lead to different visions of what is ethical [2]. Rather than one being right and one being wrong, from the functional modeling perspective both biases are needed in a functioning cognition so that its optimization function might cause it to reliably converge on some conclusion in all situations, and so that optimization function might cause it to do so in a stable and therefore selfsustaining way regardless of whatever different context it is operating in. If what a given individual's cognitive bias suggests to be true is not reliably true, then ensuring that an AGI converges on what a given individual programmer's cognitive bias suggests to be ethical is then not reliably ethical, and wherever an AGI is in a great position of responsibility and is also in the position to do harm, any measures based on such an approach must be unreliable, potentially making AGI inherently unsafe.

Looking at systems as organisms, a better solution might be to mimic the way nature defines truth when attempting to evolve a system to better achieve some target outcome. And a better solution might be to mimic the way nature adaptively changes organisms to reflect that truth, so that they self-assemble to optimize outcomes through patterns of solutions far more complex than any organism might have the capacity to use to redesign itself in a top-down process driven by its own decision-making. Whether considering the safety of the collective decision making of the human mind as the most powerful living processor on earth, or whether considering the safety of an AGI that might one day be even more powerful, reliably achieving outcomes such as safety through system design might require a similar mechanism for adaptation.

\section{Related Work}

Though others have described the concept of an Artificial General Intelligence (AGI) [3], to the 
author's knowledge the author's own work is the only model that has the potential capacity to represent all human cognitive behaviours, and the only model that objectively defines the distinguishing properties of human-level problemsolving ability (intelligence) [4] as well as defining a model by which human-like general problem-solving ability might potentially be achieved.

Though others have described the concept of a general collective intelligence factor (c factor) [5], though at least one other has defined a model for a general collective intelligence factor [6], and though others have described the concept of a collective super intelligence [7], to the author's knowledge the author's own work is the only model that defines the specific mechanisms required for a General Collective Intelligence or GCI with the potential for exponentially greater general problem-solving ability (intelligence) than any individual [innate GCI], [9]. Because of this, and because this model is so new, all of the existing literature surrounding GCI refers to the author's own published work, or to pre-prints of the author's unpublished work that are still under review.

\section{Purpose, Research Questions, and Approach}

The purpose of this paper is to justify why using functional modeling and GCI to define AI and AGI safety measures might reliably create the capacity to continuously maximize AI and AGI safety. And to justify why it might be concluded that neither AI nor AGI safety are reliably achievable without GCI.

The research questions addressed in this paper as well as the method with which they were approached is described below:

\section{Section 3: Research Question Addressed}

3.1. Is using functional modeling and GCI to define AI and AGI safety measures required to reliably create the capacity to continuously maximize AI and AGI safety?

3.2. Are AI and AGI safety reliably achievable without GCI?
Section 4: Model

4.1. Defining AI and AGI safety.

4.2. Group decision-making and AI safety.

4.3. Safety in AI as well as in AGI design.

Section 5. Method

5.1. Text

5.2. Text

Section 6. Findings

Section 7. Research Limitations

Section 8. Practical Implications

Section 9. Conclusions

\section{Model}

A full discussion of the models of individual and collective cognition is too broad for a single paper. Aspects of the model of collective cognition that are relevant to the research questions are described below.

\subsection{Defining AI and AGI Safety}

As mentioned, narrow AI is a tool, and the issue of AI safety can be represented as defining what constitutes safe operating parameters, ensuring the tool operates within those safe parameters, and ensuring humans use this tool within those parameters. From the functional modeling perspective AI are specific tools through which a human or an AGI (when implemented) might interact with the world. The issue of the safety of AGI can then be represented as ensuring AGI uses AI within safe parameters as well.

This paper represents safe operating parameters by both impact on well-being and whether or not the object of that impact is part of the user's ingroup or out-group. Taking the example of a gun, if a gun did not fire when pointed at an adversary (out-group) that would create a safety risk to the gun holder. However, if the gun fired inadvertently and struck a family member (ingroup) that would also be a safety issue. Levels of impact on well-being and levels of association with the in-group are also important. A tool might be intended to provide a non-lethal deterrent in 
which case the maximum acceptable harm is less than that resulting in a fatality, but the minimum acceptable harm is at least that required to be a deterrent. The maximum acceptable harm might also increase with the degree to which the ingroup and out-group have misaligned objectives (kinship decreases). In addition to there potentially being a maximum acceptable level of harm, there also might be a maximum acceptable level of benefit resulting from an action. For example, if a customer is given too large a quantity of a product, they might never need to return.

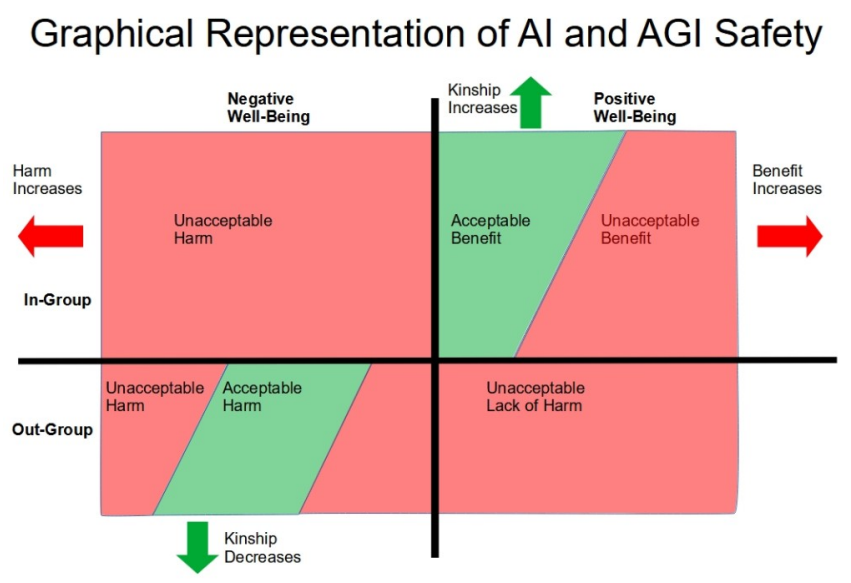

Figure 1. AI behaviour that constitutes "safe" behaviour from the perspective of the actor is in the green region. Unsafe behaviour is in the red region.

From the functional modeling perspective, any reasoning of a human or artificial system of cognition can be represented by a path through conceptual space. In addition, reasoning exists in two types, type 1 (intuitive) reasoning, and type 2 (rational methodical) reasoning [10]. Representing type 1 (intuitive) reasoning process as pattern detection, any AI can be represented as the automation of a type 1 (intuitive) reasoning

\section{Paths in Conceptual Space Representing Unsafe Associations}

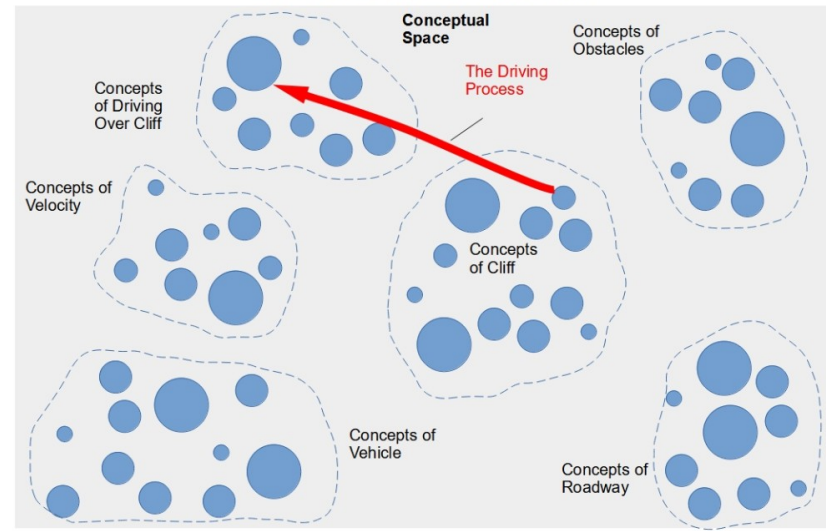

process. And any procedural software can be represented as the automation of a type 2 (methodical) reasoning process. Being reasoning processes, both can be represented as paths in conceptual space as well.

Figure 2. Reasoning paths representing the transition into the unsafe region. This transition defines unsafe reasoning actions from the perspective of well-being of the object of the action. Here the reasoning is to drive a car off a cliff.

\subsection{Group Decision-Making and AI Safety}

Centralizing or decentralization of decisionmaking determines which parties are in-groups and which parties are out-groups. In a group in which decision-making is centralized to a single individual, wherever the interests of that individual do not align with the interests of the group, the remaining members of the group become an out-group. In a group in which decision-making is decentralized in a way that inseparably binds decision-making to the collective well-being, all group members are part of the in-group. As mentioned, what actions are considered safe depends heavily on whether the object of those actions is within the in-group or within the out-group. Centralization or decentralization then heavily impacts AI and AGI safety.

Without General Collective Intelligence, the functional modeling approach suggests that groups might still have some innate general collective intelligence factor [innate GCI]. However, the nature of that collective reasoning might be fundamentally different with regards to decisions related to AI safety. The Functional Modeling Framework (FMF) [11] used to define the model of cognition this model of AGI is based on represents the human system as consisting of a number of adaptive domains. Two of these domains are the domain of the emotional system and the domain of the cognitive system. Adaptive problem-solving can occur in both of these domains. Each of these domains might have different capacities to converge on a common 
collective state within a group. Assume that emotions might converge in a group more readily than reasoning does, particularly more readily than type 2 reasoning. This is reasonable since if type 2 reasoning is like evaluating an "equation", then the values of the variables plugged into the equation, the choice of the equation itself, and the way the equation is evaluated might differ for every individual in the group. By contrast, if a given emotion is communicated, everyone in the group might feel the same way. The degree to which intuitive reasoning and rational methodical reasoning are influenced by emotion might also differ. Assuming intuition is more emotion driven than rational methodical reasoning, groups might be most reliably capable of converging on conclusions based on intuitive reasoning.

\section{Differing Relative Impact of Emotion on Intuitive and Methodical Reasoning}

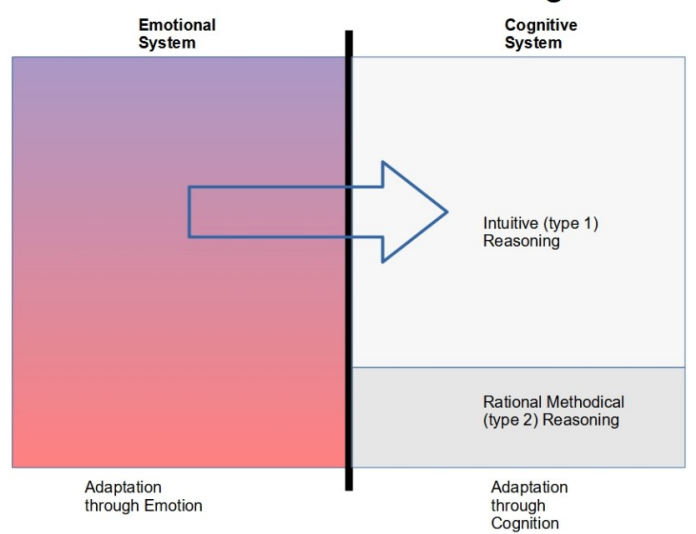

Figure 3. Emotional processes might be far more likely to drive intuitive reasoning than to drive rational methodical reasoning.

Such reasoning might centralize decision-making in groups. Because if group support for a decision is most effectively driven by emotional arguments, and if emotional arguments are most effectively made by those with an audience, then such arguments are then likely have a tendency to align with the interests of the leader, corporation, or other sub-set of the group who have an audience and that are advocating for that choice. The decisions driven by GCI however are innately decentralized. If the innate collective intelligence of groups without GCI tends to be centralized, and if centralization heavily impacts what AI actions are deemed to be safe, the AI safety decisions made by groups based on innate collective intelligence might be very different from decisions made by groups based on the artificial collective intelligence of a GCI.

Secondly, centralized decision-making might reinforce this tendency towards further centralization by concentrating resources in the hands of a centralized decision-maker. Some degree of competitive advantage or inequality is present even in natural systems, and is necessary for the natural selection that makes natural systems more fit. But this functional approach to modeling systems suggests that technology doesn't only provide competitive advantage. It radically scales human ability to store far more of this competitive advantage, and radically scales potential consumption of the resources that any competitive advantage brings. As a result, competitive advantage through technology allows a centralized owner to successfully win a greater share of resources, then to acquire more technology to acquire more resources, in an ever increasing cycle without natural physical limits.

In the case of completely centralized decisionmaking all entities in the group but one will be in the out-group. If all decision-making without collective intelligence tends to favour the interests of the decision-maker rather than optimizing collective outcomes, and if the natural tendency of decision-making without GCI is to exacerbate this tendency, trying to find a better AI capable of working safely with regards to out-groups in this centralized system of decision-making is not only misguided, but also profoundly dangerous in providing a false sense of security that distracts from the true danger of centralization.

Artificial Intelligence or any other technology isn't inherently evil. But lacking GCI, and lacking the required platform infrastructure for GCI [12], current decision-making systems might tend to ensure that technology is deployed in a centralized way that increases capacity for centralized control. As far as it is not aligned with the interests of the out-group, and as far as it results in the out-group being the majority, centralized control cannot be 
made safe. Whether that control rests in the hands of an AGI or any human, such control is by nature aligned with their interests and aligned with competing to increase their interests, rather than to increase collective well-being.

Systems of decentralized cooperation have the potential to be far safer while also providing powerful competitive advantage to the group over those trying to compete as individuals. This competitive advantage comes from cooperating to decrease consumption, and cooperating to maximize collective well-being [13].

\subsection{Safety in AI as well as AGI Design}

From a functional modeling perspective, designing AI for safety begins with creating the capacity to represent any and all reasoning processes in conceptual space, where those processes might be implemented using AI, so that the projected fitness of those processes in achieving impact on the well-being of in-groups or out-groups might be determined in different contexts. The representation of reasoning in conceptual space is a semantic representation of reasoning. Once AI processes are represented semantically, ensuring AI safety becomes a matter of preventing certain associations from being made. That is, preventing the AI from taking certain paths through conceptual space by designing functionality to prevent any training data from leading to the detection of unsafe patterns.

An AGI on the other hand might well eventually have the ability to design itself. So the important considerations with AGI are both individual agency and how that multiple entities with that individual agency combine to produce group decision-making behaviour. As mentioned, group decision-making processes that lack GCI, whether involving humans, AGI, or both, centralize decision-making so that AI design might be inherently unsafe for most.

As for individual agency and AI safety, both humans and AGI must be provided with incentives to safe behaviour, and consequences to unsafe behaviour. If safe or unsafe behaviour can be represented as paths in conceptual space, and if consequences or incentives can be represented in the well-being space of the individual human or AGI cognitive system, then the capacity to maximize outcomes representing incentives or consequences might be created through networks of cooperation [13]. Incentives or consequences might then be increased to the point that they are sufficient to reliably deter unsafe behaviour.

\section{Method}

The research question was addressed using the methods summarized in table 2 .

Table 2. Components of cognitive process execution.

\begin{tabular}{|c|c|}
\hline Research Question & Method \\
\hline $\begin{array}{l}\text { 1. Is using functional } \\
\text { modeling and GCI to } \\
\text { define AI and AGI } \\
\text { safety measures } \\
\text { required to reliably } \\
\text { create the capacity to } \\
\text { continuously } \\
\text { maximize AI and } \\
\text { AGI safety? }\end{array}$ & $\begin{array}{l}\text { 1. The requirements } \\
\text { for identifying all } \\
\text { unsafe behaviours } \\
\text { were considered to } \\
\text { assess whether doing } \\
\text { so might require } \\
\text { functional modeling. } \\
2 \text {. The requirements } \\
\text { for maximizing the } \\
\text { safety of all AI } \\
\text { behaviours were } \\
\text { assessed to } \\
\text { determine whether } \\
\text { GCI was required to } \\
\text { continuously } \\
\text { maximize AI and } \\
\text { AGI safety. }\end{array}$ \\
\hline $\begin{array}{l}\text { 2. Are AI and AGI } \\
\text { safety reliably } \\
\text { achievable without } \\
\text { GCI? }\end{array}$ & $\begin{array}{l}\text { 3. The measures } \\
\text { required to reliably } \\
\text { achieve AI and AGI } \\
\text { safety through } \\
\text { external means of } \\
\text { control were } \\
\text { identified. } \\
\text { 4. The measures } \\
\text { required to reliably } \\
\text { achieve AI and AGI }\end{array}$ \\
\hline
\end{tabular}


safety through

internal means of

control were

identified.

5. The feasibility of successfully

implementing those

control measures

without GCI was

assessed.
5.1. Identifying Unsafe Behaviours and Functional Modeling

Define cognitive well-being as the fitness of the cognitive system to perform all of its functions. Assume any reasoning that might be executed by any actor can be represented by a path in the conceptual space of that actor, where the action is constituted by use of AI in some way. Assume any reasoning by which the impact can be observed might be represented as paths in the conceptual space of the object of those actions. Then whether that behaviour is safe or unsafe is determined by whether the point in the well-being space of the object of that action, corresponding to the final concept in the conceptual space of the object of that action, is within the safe region of well-being behaviour.

\section{Mapping Reasoning to Safety}

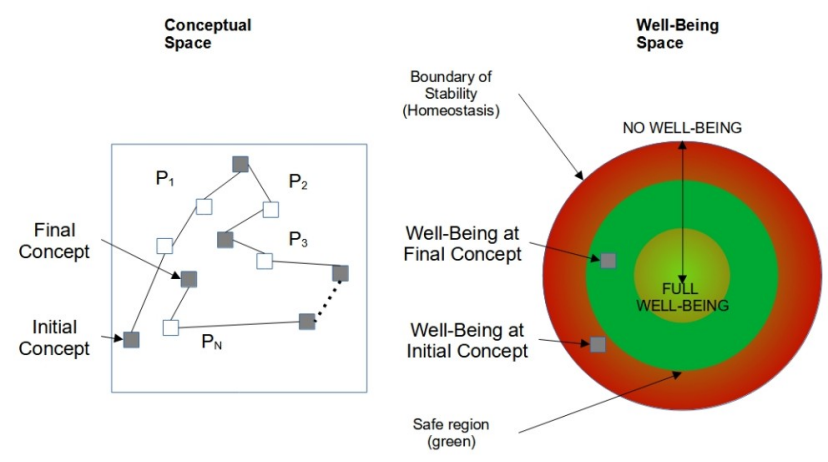

Figure 3. Modeling AI safety by mapping reasoning of the actor to the safety of the object. From the perspective of the actor safe actions are those for which well-being ends in the green region.

\subsection{Maximizing Safety and GCI}

Multiple reasoning paths through conceptual space to solve the same problem might be possible. These reasoning paths might differ in their fitness to achieve targeted outcomes and therefore their impact on cognitive well-being. By assessing all available reasoning paths, it might be possible to increase impact on any targeted outcome.

\section{Multiple Reasoning Paths to Same Conclusion in Conceptual Space}

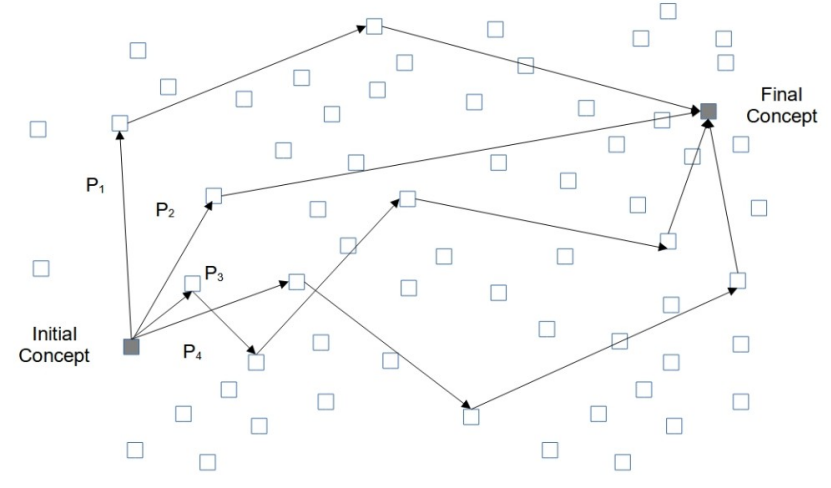

Figure 4. Maximizing outcomes by identifying all reasoning paths and comparing outcomes.

Once it is possible to identify all AI actions as safe or unsafe in terms of whether or not they terminate in the safe zone of well-being of the object, as defined by the in-group or out-group relationship of the actor to that object, then it might be possible to increase the safety of AI actions by comparing all possible AI actions where those AI actions solve the same problems (have the same concepts as inputs and the same concepts as outputs).

5.3. Methods for Achieving External Control of an Artificial General Intelligence or of Humans in their Use of AI 
External control of any intelligent actor, whether human or AGI, can take the form of negative coercion, or positive incentivization.

\section{Negative Coercion}

From a functional modeling perspective, human beings consist of the physical, emotional, cognitive, and consciousness systems. Each of these systems has a well-being space. The vectors through which human beings might be negatively coerced to avoid intentionally using AI in unsafe ways involve the threat of negative consequences to well-being in these systems. These consequences might be represented for example by threat of fines, public censure, imprisonment, or other punishments. The vectors through which human beings might be negatively coerced to avoid unintentionally using AI in unsafe ways involve conveying an understanding that particular uses are unsafe in particular contexts.

An AGI might only implement the cognitive system, and therefore might only have the vector of cognitive well-being through which it might be coerced. Cognitive well-being is determined by the ability (fitness) of the cognitive system to execute all its cognitive functions. This cognitive well-being might be negatively impacted by giving the cognitive system difficult problems that must be solved before unsafe reasoning processes can be engaged, or before any reasoning at all might be resumed. As an example in the case of an AGI, the threat of simply being turned off within milliseconds of engaging in unsafe reasoning, and the problem of having those milliseconds worth of answers removed from the AGI's conceptual space, are then problems that the AGI must solved before undertaking to figure out how to conduct an unsafe action. Solving those problems then would need to be conducted in milliseconds. In the case of a human actor, true AGI functionality might likely require GCI in its design and implementation [14]. GCI could then provide individuals access to their own personal AGI while also building in relationships with every other individual AGI, where those relationships might be broken by unsafe use. In this way individual's contemplating unsafe use might be faced with solving the problem of cheating potentially far greater than 7.5 billion relationships, several for each person on earth.

\section{Incentivization}

The vectors through which human beings or AGI might be positively incentivize to use AI in safe ways involve the potential to increase well-being. Any available benefits might be leveraged in this way.

\subsection{Methods for Achieving Internal Control of an Artificial General Intelligence or of Humans in their Use of AI}

Internal control for any intelligent actor, whether human or AGI, can take the form of positive guidance towards wisdom, or negative guidance through indoctrinization. In the case of humans, having a consciousness system as well, internal control can take the form of positive guidance towards enlightenment, or negative guidance through attachment.

\section{Indoctrinization and Attachment}

As mentioned, one mechanism of internal control is making the human or AGI believe so strongly that harming humans is always wrong, that it refuses to do so. In other words to indoctrinate that individual entity. Effective indoctrination requires providing a sufficient number of effective examples to train the actor's intuition with the desired conclusion in the targeted subject. Maximizing the effectiveness of those examples in order to maximize effectiveness of indoctrination might include mapping the conceptual space of each individual actor, and assessing the impact of different reasoning processes on all actors with similar graphs in conceptual space, to determine the most effective actions. However, indoctrization is inherently a centralized process in that it is driven by a centralized actor. Indoctrinization, and the AI actions permitted by an actor's indoctrinization, cannot therefore be reliably made safe.

Having consciousness, the consciousness of any individual human being might be attached to certain awarenesses. Wherever these attachments cannot be let go they are a mechanism for 
coercing humans into safe AI use. Again however, wherever such coercion is centralized, such coercion cannot be made safe, and therefore the AI actions permitted by an actor's coercion, cannot therefore be reliably made safe.

\section{Wisdom and Enlightenment}

In the Functional Modeling Framework (FMF) [11] used to represent the human system, consciousness is a separate subsystem. Just as intelligence is a measure of capacity for adaptive problem-solving ability in the cognitive domain, enlightenment is a measure of adaptive problemsolving ability in the consciousness domain. Where the cognition navigates a conceptual space, the consciousness navigates a space of awarenesses or an awareness space. Just as the level of intelligence is represented by the volume of conceptual space that can be navigated per unit of time in order to reach a concept that solves a problem in the cognitive domain, the level of enlightenment is represented by the volume of awareness space that can be navigated per unit time to reach an awareness that solves a problem in the consciousness domain. As an example, a more enlightened consciousness might simply have the capacity to draw itself to whatever awareness provides well-being in a given context. A less enlightened consciousness might lack the capacity to draw itself from whatever awareness that is harming its well-being.

In addition to innate intelligence and innate awareness, problem-solving ability in the cognitive domain might be increased by providing guidance that increases problem-solving ability, that is, providing guidance so that the cognitive systems becomes more wise. And problemsolving in the consciousness domain might be increased by providing guidance so that the consciousness is more enlightened. Because AGI and GCI as currently conceived only implement the cognitive domain, enlightenment only applies to safety in the way individual humans use AI.

Enabling humans to become enlightened enough to see themselves as part of a larger cooperating whole could potentially remove the incentive to prioritize individual needs over those of others. This would require that individuals actually be part of a larger cooperating whole. Creating this cooperation might require implementing General Collective Intelligence society-wide to create complex networks of cooperation that constrain behaviours to those resulting in mutual benefit [13]. According to the model of GCI, lacking the decentralization that only GCI can provide, civilizations might currently be run by decisionmaking systems that align decision-making with the interests of a centralized subset of decisionmakers acting in competition with others, where the goal is to in essence win more decisionmaking power. As mentioned, decision-making in any centralized system, whether AI or human, cannot be assured to be safe.

\subsection{Feasibility of Achieving and Maintaining External or Internal Control without GCI}

Cognitive well-being might be negatively impacted through external means by giving the cognitive system unsolvable problems that must be solved before unsafe reasoning processes can be engaged or continued. And cognitive wellbeing might be positively impacted through external means by giving the cognitive system benefits as a result of safe AI use, where the problem of removing those benefit can't reliably be solved by any other entity, and therefore can't reliably be removed.

Since an AGI might have the capacity for exponentially greater general problem-solving ability than any individual, any problem that is unsolvable to a human and that is given to an AGI as a deterrent, or incentive, might potentially be solvable by that or another AGI and therefore not suitable for either purpose. GCI is required to exponentially increase problem-solving ability so that it might reliably be greater than that of an AGI, thereby making deterrents and benefits unremovable. In this way, achieving external deterrents or incentives without GCI might not be reliably achievable.

For humans or AGI, cognitive well-being might be impacted positively through internal means by 
instilling wisdom, or negatively through internal means by indoctrination. In the case of humans, conscious well-being might be impacted positively through internal means by enlightenment, or negatively through internal means by attachment. Problems of higher order complexity than humans can reliably define or solve require an AGI or GCI to solve. The problem of guiding the conscious awareness of every individual human, or the problem of educating the intelligence of every individual human or AGI, is likely a higher order problem. Solving this problem with AGI inherently has the potential to be unsafe if AGI is itself not safe. Therefore this problem must be solved with GCI.

\section{Findings}

Determining whether any AI behaviour is safe requires the ability to model all AI behaviour and to model the safety of that behaviour in all contexts. And maximizing safety in the way AI is used, or maximizing safety in the actions an AGI takes, requires the exponential increase in general problem-solving ability that outside of AGI appears to be possible only through a GCI. However, safety in complex systems is a problem nature has already solved, since in any organism, the countless interactions between its cells, are overwhelmingly safe. And nature has proved that solution over billions of years. The functional modeling approach represents safety of any processes in any domain as occurring through adaptive problem-solving solving processes in that domain that share common design elements enabling them to reliably converge on maximum fitness to execute all functions in that domain. GCI implements this adaptive problem-solving to optimize collective outcomes.

Because functional modeling is required for GCI, and GCI is required for AI and AGI safety, then functional modeling is required for AI safety as well. Because the speed and scale with which an AGI or with which groups of human-beings can define new uses of AI might be exponentially greater than the speed or scale at which any individual or group without GCI might assess the safety of those actions. AI and AGI safety might then not be reliably achievable without GCI.

Table 3. Research Findings.

\begin{tabular}{ll}
\hline Research Question & Findings \\
\hline $\begin{array}{l}\text { 1. Is using functional } \\
\text { modeling and GCI to } \\
\text { define AI and AGI }\end{array}$ & $\begin{array}{l}\text { 1. Pending } \\
\text { experimental } \\
\text { confirmation the } \\
\text { safety measures } \\
\text { required to reliably } \\
\text { create the capacity to } \\
\text { continuously }\end{array}$ \\
$\begin{array}{l}\text { maximize AI and AGI } \\
\text { safety? }\end{array}$ & \\
\hline $\begin{array}{l}\text { 2. Are AI and AGI } \\
\text { safety reliably } \\
\text { achievable without }\end{array}$ & $\begin{array}{l}\text { 2. Pending } \\
\text { experimental } \\
\text { GCI? }\end{array}$ \\
& $\begin{array}{l}\text { confirmation the } \\
\text { answer appears to } \\
\text { be no. }\end{array}$ \\
\hline
\end{tabular}

\section{Research Limitations}

Though models of a number of proposed GCI solutions have been defined in agriculture, health care, and other fields, and though conceptual case studies of these solutions have demonstrated the potential for the exponential increase in impact on outcomes as suggested might be achievable in this paper, GCI has not yet been implemented. And though some elements of the conceptual space have been demonstrated through simulation [4], the conceptual space has not yet been fully implemented either. As a consequence, many details inevitably remain to be clarified.

Furthermore, as described elsewhere [15], GCI is a complex system that must likely in effect be grown through initiating a self-assembling process that adapts in any way required to maximize collective outcomes rather than being developed in a top-down way that can become tightly coupled with centralized interests. Defining a simple functional model of GCI alone is not sufficient to implement GCI. An understanding of the self-reinforcing networks of cooperation and 
hierarchy of their deployment that is required [13], an understanding of the application of GCI to the customization of products and services deployed in those networks [16], an understanding of the platform required to execute processes with a GCI [12], as well as an understanding of the application of GCI to research, design [17] and all the other processes in the business life-cycle might be required. In the same way that defining a simple functional model of the human system as containing a heart to pump blood, lungs to oxygenate blood and remove carbon dioxide, as well as containing other functional components is not sufficient to clone a human being. However, the process of understanding how to implement a GCI must begin with an understanding of the functional components required.

\section{Practical Implications}

AI is often a black box. Understanding it's inner workings might not be achievable. AI inherently has the potential to be unsafe if it's use cannot be constrained, if its use cannot easily be verified, and if humans or AGI making use of it can't be incentivized or constrained to use it safely. For centralized deployments of AI in which the interests of the majority are not aligned with the interests of the entity owning the AI, AI might inherently have the potential to be unsafe in an increasing number of circumstances as the ability of the majority to constrain the behaviour of that centralized entity decreases.

Though measures that might impact cognitive well-being might be used to incentivize the safe use of AI and discourage the unsafe use of AI, for an AGI that could rapidly become smarter than humans, this is a dangerous strategy if the AGI is given centralized control of functions that are critical for human existence. Again, the best way to ensure safety is to decentralize this control over multiple AIs, each of which are controlled by a separate entity that does not answer to the same command structure so they can't be coerced to command harm to serve that centralized interest. For example, if a single AGI ran critical functions in the power grid, and critical functions in the transport and financial systems, and they were all controlled by a single government, that government would have the ability to shut off the power for a rebellious region so no food that requires refrigeration would remain fresh past 3 days, as well as having the ability to shut off the transport system so no food could be delivered, and to shut off the financial system so no food could be purchased by any alternative means. Such a government would have in effect weaponized AGI, thereby making it inherently unsafe. The point at which a centralized AI becomes a centralized AGI potentially can't be predicted. Therefore this scenario can't be prevented with the centralized deployment of AI and AGI.

An external measure of control for a AGI would be ensuring that it is not given any critical functions, and that all functions it is given control over could only operate within safe parameters. This would be the equivalent of ensuring the AGI would only be given plastic scissors to cut with. Competitive pressures and sheer inconvenience however, might ensure that such regulations would be gradually relaxed and/or ignored.

\section{Conclusions}

While virtually all work on AI safety has focused on how to control AI through various top down internal or external means according to what is right or wrong by some AI researcher, or AI research donor's perspective, all top-down methods are centralized, and therefore have the potential to be inherently unsafe. However, nature has already solved the problem of AI safety in the decentralized way it designs all organisms so that their functional components are safe. In nature's safety framework, one component, such as a cell, is bound by countless cooperative relationships which form a network of cooperation that constrict that component's behaviour. This solution has been developed and proven over billions of years. Designing a bottom-up process in which the use of AI and AGI self-assemble in ways that maximize human safety might be a problem of higher order complexity than any 
individual can define or solve, and therefore might require General Collective Intelligence to replicate the same kinds of networks of cooperation that

\section{Acknowledgements.}

Thanks to Julian Talev for countless discussions on the topic.

\section{References}

1 Journal article: J. Hernández-Orallo, "AI Safety Landscape From short-term specific system engineering to long-term artificial general intelligence," 2020 50th Annual IEEE/IFIP International Conference on Dependable Systems and Networks Workshops (DSN-W), Valencia, Spain, 2020 , pp. 72-73, doi: 10.1109/DSNW50199.2020.00023.

2 Pre-print: Williams, A. E. (2020, November 17). Bridging the Left - Right Political Divide with Artificial Intelligence and Collective Intelligence. https://doi.org/10.31730/osf.io/y65x4

3 Book Chapter: Williams A.E. (2020) A Model for Artificial General Intelligence. In: Goertzel B., Panov A., Potapov A., Yampolskiy R. (eds) Artificial General Intelligence. AGI 2020. Lecture Notes in Computer Science, vol 12177. Springer, Cham. https://doi.org/10.1007/978-3-03052152-3 38

4 Pre-print: Williams, A. E. (2020, July 11). Human Intelligence and General Collective Intelligence as Phase Changes in Animal Intelligence. https://doi.org/10.31234/osf.io/ dr8qn

5 Journal article: Woolley, Anita Williams; Chabris, Christopher F.; Pentland, Alex; Hashmi, Nada; Malone, Thomas W. (29 October 2010). "Evidence for a Collective Intelligence Factor in the Performance of Human Groups". Science. 330 (6004): 686688. Bibcode:2010Sci...330..686W. doi:10.1126/science.1193147. PMID 20929725. S2CID 74579.

6 Journal article: Krafft, P.M. (2019), A Simple Computational Theory of General Collective Intelligence. Top Cogn Sci, 11: nature uses, so that AI safety is reliably achievable.

\section{4-392.}

https://doi.org/10.1111/tops.12341.

7 Book: Malone, T. W. (2018). Superminds: The surprising power of people and computers thinking together.

8 Pre-print: Williams, A. E. (2020, December 2). General Collective Intelligence vs the Innate Collective Intelligence Factor. https://doi.org/10.31730/osf.io/kp3x8

9 Conference: The Relationship Between Collective Intelligence and One Model of General Collective Intelligence, Andy E. Williams, Computational Collective Intelligence, 11th International Conference, ICCCI 2019, Hendaye, France, September 4-6, 2019, Proceedings, Part II, Pages 589600

10 Book: Kahneman, Daniel. Thinking, Fast and Slow. New York: Farrar, Straus and Giroux, 2011.

11 Pre-print: Williams, A. E. (2020, April 16). A Human-Centric Functional modelling Framework for Defining and Comparing Models of Consciousness and Cognition. https://doi.org/10.31234/osf.io/94gw3

12 Pre-print: Williams, A. E. (n.d.). The Peer to Peer Social Fabric as a Platform for General Collective Intelligence. Retrieved from osf.io/preprints/africarxiv/qbxfr

13 Pre-print: Williams, A. E. (2020, December 16). Discovering and Implementing Self-Sustaining Networks of Cooperation with General Collective Intelligence. https://doi.org/10.31730/osf.io/ $\underline{\text { safxk }}$

14 Pre-print: Williams, A. E. (2020, August 17). Does Creating an Artificial General Intelligence Require General Collective Intelligence in Order to be Reliably Achievable?.

https://doi.org/10.31730/osf.io/3q72c

15 Pre-print: Williams, A. E. (2020, November 11). General Collective Intelligence and the Transition to Collective 
Super-Intelligence. https://doi.org/10.31730/ osf.io/tacyq

16 Pre-print: Williams, A. E. (n.d.). Individualization of Products and Services with Artificial General Intelligence and General Collective Intelligence. Retrieved from osf.io/preprints/africarxiv/gd5mt

17 Pre-print: Williams, A. E. (2020, December 18). Increasing Discovery in Research, Design, and Other Processes with Artificial General Intelligence and General Collective Intelligence.

https://doi.org/10.31730/osf.io/gz385 